\title{
Experimental demonstration of distance measurement with a femtosecond frequency comb laser
}

M. Cui

m.cui@tudelft.nl

\section{R. N. Schouten}

\section{N. Bhattacharya}

\section{S.A. van den Berg}

\author{
Optics Research Group, Department of Applied Sciences, Technical University Delft, Lorentzweg 1, \\ 2628 CJ Delft, The Netherlands \\ Optics Research Group, Department of Applied Sciences, Technical University Delft, Lorentzweg 1, \\ 2628 CJ Delft, The Netherlands \\ Optics Research Group, Department of Applied Sciences, Technical University Delft, Lorentzweg 1, \\ 2628 CJ Delft, The Netherlands \\ NMi Van Swinden Laboratory, Thijsseweg 11, 2629 JA Delft, The Netherlands
}

We experimentally demonstrate that a stabilized femtosecond frequency comb can be applied as a tool for distance measurement in an interferometric scheme. A proof of principle of this method, as proposed by Ye [5], is provided by measuring a displacement of about $15 \mathrm{~cm}$ in air and comparing it to a reference value from a calibrated laser interferometer. The experiment shows that the new scheme easily achieves an accuracy better than one optical fringe. [DOI: 10.2971/jeos.2008.08003]

Keywords: Ultrashort pulse, cross-correlation, frequency comb, interferometry

\section{INTRODUCTION}

Since the invention of the laser numerous applications of lasers in the field of length and distance measurement have been developed. Using traditional techniques such as classical interferometry, accuracy at the nanometer level can be reached. These techniques are generally incremental and are applied to measure displacement. To measure absolute length, the value of the measured length has to be known beforehand with sub-wavelength accuracy, because of the ambiguity of the interferometric measurement results. In 1990s, multiplewavelength interferometry was implemented to measure absolute lengths. By appropriate choice of wavelengths a longer synthetic wavelength is generated, leading to relaxed requirements on the initial value of the distance to be measured [1]. The use of various optical frequencies makes the system fairly complex because a number of sources is needed. Alternatively, frequency sweeping techniques can be applied for measuring absolute distance [2]. In 2000, a measurement technique using the frequency comb from a femtosecond mode locked laser was developed and a measurement of distances upto $240 \mathrm{~m}$ was reported by Minoshima et.al. [3]. In this experiment, the femtosecond laser is applied as an advanced modulator, with the repetition rate of the laser being the modulation frequency. Distance measurement is based on phase detection of higher harmonics of the repetition frequency and takes place in the radio frequency domain. In 2006, another technique using dispersive interferometry was developed [4]. In this scheme, a femtosecond laser was used as a white light source and the delay in the optical path of two consecutive ultrashort pulses was measured by means of spectral analysis of the interferometer signal. An accuracy of $7 \mathrm{~nm}$ was achieved, with a measurable distance up to 1 meter. In 2004, a novel scheme for measuring long distances in vacuum with a stabilized femtosecond frequency comb was proposed by Ye [5]. The scheme is based on optical interference between individual pulses in a Michelson type interferometer. Interference is possible because the carrier-envelope stabilized pulses have a fixed phase relation with respect to each other. This scheme may be viewed as an extension of multi-wavelength interferometry, with the phase-stabilized mode-locked laser as a multi-wavelength source. In this paper we provide an experimental proof of principle of this method by measuring a short displacement $(15 \mathrm{~cm})$ in air with a phase-locked fs laser and comparing the result to a calibrated laser interferometer.

\section{MEASUREMENT PRINCIPLE}

A frequency comb is generated from a phase-stabilized modelocked femtosecond laser [6]-[8]. The periodic train of pulses emitted from the laser has its counterpart in the frequency domain as a comb of equidistant modes with a mutual separation equal to the repetition frequency $f_{r}$. The difference between the group velocity and phase velocity gives rise to a pulse to pulse phase shift $\Delta \phi$ between the carrier wave and the envelope. This phase shift results in an offset frequency $f_{0}$, generally referred to as the carrier-envelope offset (CEO) frequency. This is illustrated in Figure 1. The CEO and the repetition frequency are related by $f_{0}=(\Delta \phi / 2 \pi) f_{r}$.

Both $f_{r}$ and $f_{0}$ are stabilized to a reference value. As a result of stabilizing $f_{r}$ and $f_{0}$, the distance between the pulses $l_{p p}$ is fixed and the CEO is stabilized. 


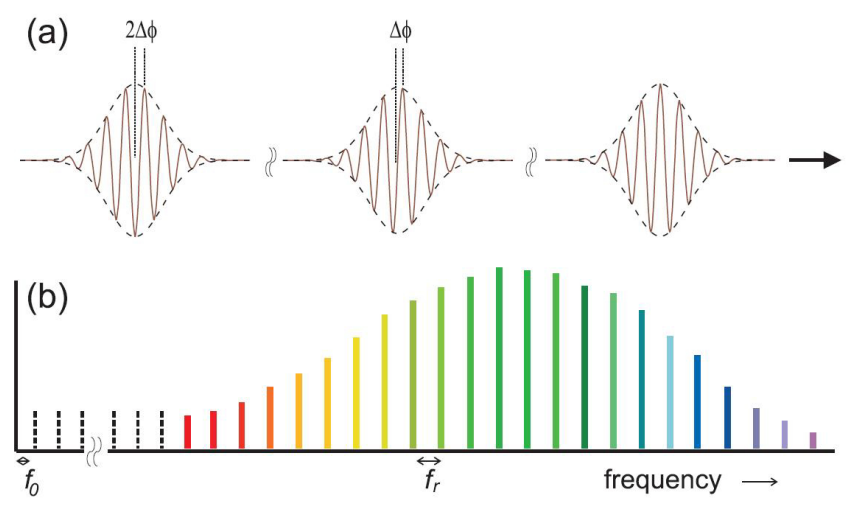

FIG. 1 a) Illustration of the carrier-envelope phase shift in the time domain. A pulse to pulse phase shift $\Delta \phi$ is observed. b) Corresponding optical frequency spectrum with $f_{0}$ the offset frequency due to the carrier-envelope phase shift, and $f_{r}$ the pulse repetition rate.

For distance measurement the pulse train is sent into a Michelson interferometer consisting of a measurement arm and a reference arm. A coherence maximum in the interferometer output is obtained once the path length difference between the arms is a multiple of $l_{p p}$. This can be accomplished by changing the cavity length, and thus the interpulse distance [5]. An alternative method is to apply a calibrated delay line to tune the path length difference to a multiple of the interpulse distance. If the range of the delay line exceeds $l_{p p} / 2$, spatial overlap between the pulses can always be accomplished. The interpulse distance $l_{p p}$ can be viewed as the synthetic wavelength, corresponding to the beat frequency of adjacent modes, and thus to the repetition rate. The non-ambiguity range of the distance measurement is determined by $l_{p p}$ and is of the order of $1 \mathrm{~m}$ for most fs laser sources. Hence the required initial value of the distance to be measured, can be easily determined, e.g. with a time-of-flight measurement.

In our setup we use a delay line to accomplish temporal overlap of the pulse trains coming from both arms of the interferometer. Subsequently, detection of the interference takes place by measuring a cross-correlation function. The crosscorrelation function is obtained by scanning the delay line with a piezo-element. The position of the center of the envelope of the cross-correlation function can be found by curve fitting. With this method the position of the mirror in the measurement arm can be measured absolutely with respect to a reference position in a non-incremental way. In this paper we experimentally verify this measurement principle by measuring a short displacement (equal to $l_{p p}$ ) and comparing the results with a counting laser interferometer. The accuracy, limitations and possible improvements to this method will be discussed.

\section{EXPERIMENTAL METHOD}

A schematic of the experimental setup is shown in Figure 2. The measurements are carried out with a mode-locked Ti:Sapphire laser with a pulse duration of 40 femtosecond. Both the repetition frequency and the carrier-envelope-offset frequeny are locked and referenced to a cesium clock. The

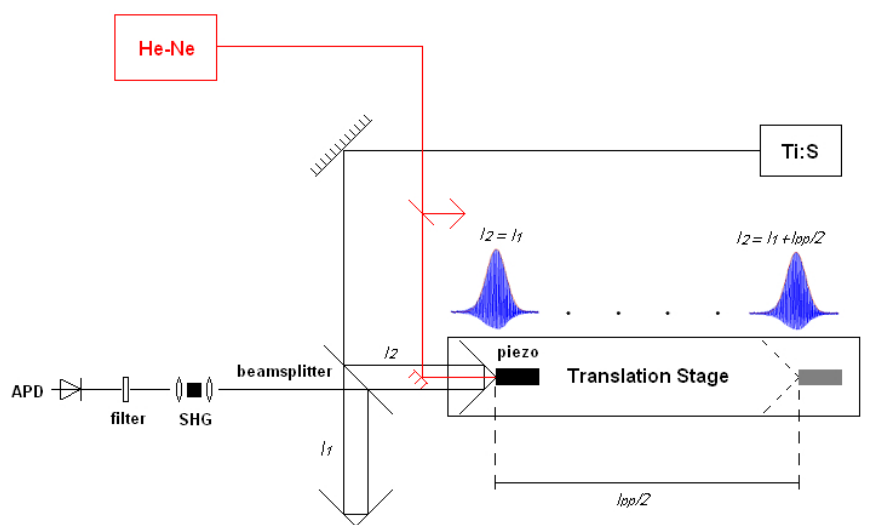

FIG. 2 Schematic of the experimental setup. A pulse train is sent into an interferometer and cross-correlation functions are measured at two positions of the translation stage, separated by a distance $l_{p p} / 2$. Simultaneously, the displacement is measured with a Zeeman-stabilized laser interferometer. The incoming and reflected beams propagate in a plane perpendicular to the plane of the drawing at different heights.

output wavelength of the pulse is centered at $820 \mathrm{~nm}$ with a bandwidth of $20 \mathrm{~nm}$. The repetition rate is approximately $1 \mathrm{GHz}$, corresponding to a cavity length of the laser of about $15 \mathrm{~cm}$. The cavity length can be subtly adjusted and thus the repetition rate of the laser. The offset frequency is fixed at $f_{0}=180 \mathrm{MHz}$. The pulses from the laser are split into two at the beam splitter. One part of the pulse goes into the non-varying arm of the interferometer with length $l_{1}$ and is reflected by a retro-reflector. The other part of the pulse goes into the other arm with length $l_{2}$. The length of this arm can be varied with a translation stage and a piezo-element. During the measurement, the retro-reflector is translated back and forth with a frequency of $1 \mathrm{~Hz}$ with a saw-toothed voltage. A maximum movement of around 55 microns is achieved. The two reflected beams coincide with each other and are focused inside a Barium Borate Crystal (BBO). A filter is used to block the original infrared light. The second harmonic signal generated in the crystal is focused onto an avalanche photodiode (APD). The intensity of the second harmonic signal is measured with an oscilloscope.

The experiment is done in the following manner. First the translation stage is set such that $l_{1}$ and $l_{2}$ are equal and a crosscorrelation function is measured (position $A$ ). Then the stage is translated such that $l_{2}$ is close to $l_{1}+l_{p p} / 2$ (position $B$ ). At this position another cross-correlation function is measured. Since the translation stage has a finite step size, it is not possible to position the stage such that the maximum value of the cross-correlation function occurs at exactly the same piezo voltage as at position $A$. Gaussian functions are fit through the profiles of the two recorded correlation patterns. By comparing the maximum of the second cross-correlation with the first one, a correction term $\delta_{c}$ is obtained. Since the distance between two adjacent teeth is one wavelength, a scale is available and the real displacement between the two peaks of the correlation functions can be calculated. The cross-correlation functions at both positions are shown in Figure 3.

The pulse-to-pulse distance $l_{p p}$ is calculated from the repetition frequency by $l_{p p}=v_{g} / f_{r}$, where $v_{g}$ is the group velocity 


\begin{tabular}{|c|c|c|c|c|}
\hline $\begin{array}{c}l_{p p} / 2 \\
(\mathrm{~nm})\end{array}$ & $\begin{array}{c}\text { Displacement } \\
\delta_{c} / 2(\mathrm{~nm})\end{array}$ & $\begin{array}{c}\text { Measured distance } \\
\text { frequency comb(nm) }\end{array}$ & $\begin{array}{c}\text { Reference distance } \\
\text { He-Ne laser }(\mathrm{nm})\end{array}$ & $\begin{array}{c}\text { Difference } \\
(\mathrm{nm})\end{array}$ \\
\hline \hline $147,765,420$ & +396 & $\begin{array}{c}147,765,816 \\
\pm 112\end{array}$ & $147,765,593$ & +223 \\
\hline $147,765,493$ & -571 & $\begin{array}{c}147,764,922 \\
\pm 250\end{array}$ & $147,764,996$ & -74 \\
\hline $147,765,566$ & -836 & $\begin{array}{c}147,764,730 \\
\pm 339\end{array}$ & $147,764,815$ & -85 \\
\hline \hline $147,779,205$ & -261 & $\begin{array}{c}147,778,944 \\
\pm 224\end{array}$ & $147,779,054$ & -110 \\
\hline $147,779,278$ & -124 & $\begin{array}{c}147,779,154 \\
\pm 276\end{array}$ & $147,779,464$ & -310 \\
& & $\begin{array}{c}147,779,479 \\
\pm 174\end{array}$ & $147,779,383$ & +96 \\
\hline $147,779,351$ & +128 & & & \\
& & & & \\
\hline
\end{tabular}

TABLE 1 Measurement results using the two lasers. The average of ten measurements of the frequency comb is shown along with the standard deviation below it.

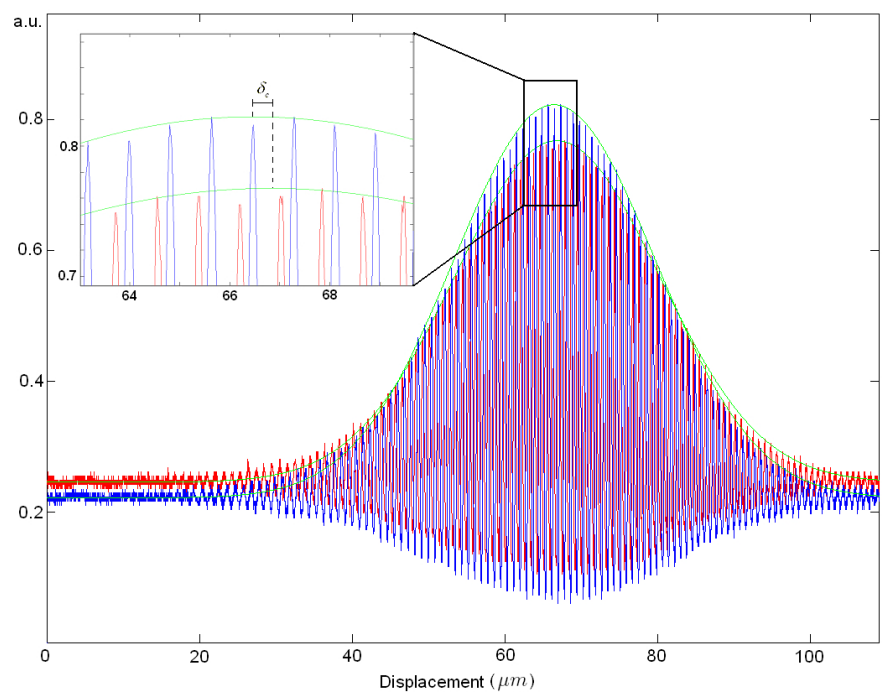

FIG. 3 Comparison of the two cross-correlation functions at position $A$ (blue) and $B$ (red). Note the small shift between these two. This shift is determined by Gaussian curve fitting of both measurements.

in air:

$$
v_{g}=c\left(n-\lambda \frac{d n}{d \lambda}\right)^{-1}
$$

Here, $\lambda$ is the wavelength, $n$ is the refractive index of the central wavelength of the pulse from the laser in air $[9,10]$ and $c$ is the speed of light in vacuum. Then, the real displacement $D$ of the retro-reflector is

$$
D=l_{p p} / 2+\delta_{c} / 2
$$

The displacement $D$ is measured independently with a calibrated He-Ne laser interferometer. The fluctuation of the temperature in the laboratory was controlled to be less than $0.1^{\circ} \mathrm{C}$, and also the air pressure change was measured to be within $1 \mathrm{hPa}$ during the measurement. Therefore the uncertainty on the He-Ne laser was dominated by the $40 \mathrm{~cm}$ dead path length and is estimated to be around $50 \mathrm{~nm}$ as specified in the manual. This value is much smaller than the fluctuations seen in the measurements done with the Ti:S laser. A position sensitive detector is used for the alignment of the beams parallel to the translation direction of the stage, leading to a negligible alignment error.

\section{RESULTS AND DISCUSSION}

The whole experiment including the control measurement has been carried out for six independent values of the repetition frequency $f_{r}$. The first three are carried out with the temperature stablized at $20.17^{\circ} \mathrm{C}$ and air pressure at $1011.2 \mathrm{hPa}$. The last three measurements took place at modified environmental conditions given by $T=20.11^{\circ} \mathrm{C}$ and $p=1025.2$ $\mathrm{hPa}$. The group velocities are calculated by using the updated Edlen equation [9, 10], and are $299710681 \mathrm{~m} / \mathrm{s}$ and 299709555 $\mathrm{m} / \mathrm{s}$ for the two sets of environmental conditions, respectively. Each of the measurements by the frequency comb is repeated ten times and the results are shown in Table 1. From Table 1 it can be seen that the distance measurements using the femtosecond laser agree with the reference measurement using the He-Ne laser within less than half a wavelength. The standard deviation of the measurements is attributed to the timing jitter in the detection system which causes the crosscorrelation function to shift from time to time. For the time scales which play a role in this measurement the timing jitter of the frequency comb laser can be neglected in comparison to that present in the piezo. The piezo-element is powered by a function generator and a high voltage power supply. The main part of the jitter is suspected to be caused by drifts in the high voltage power supply and the resulting vibration of the piezo on the translation stage.

\section{CONCLUSION}

We have experimentally demonstrated that an optical frequency comb can be applied for interferometric measurement of displacement. For a short displacement of $15 \mathrm{~cm}$ the results agree within less than half a wavelength with a calibrated reference interferometer. Improvement of the stability of the setup and especially of the piezo-element, is expected to improve the length measurement results. In this experiment we determined the displacement from the curve fitting of the envelope. However, once the jitter on the cross-correlation func- 
tion is reduced, one can easily go one step further by using the sub-structure of the cross-correlation function to improve accuracy. This can be considered as normal interferometry with the carrier-wave. In other words, the ambiguity of the measurement is taken away by measuring the position of the envelope and the highest accuracy is then obtained by conventional interferometry of the carrier wave.

\section{ACKNOWLEDGEMENT}

This research was carried out in the framework of an Industrial Partnership Program on Optical Metrology of FOM, the Dutch Foundation for Fundamental Research on Matter. The authors acknowledge fruitful discussions with Joseph Braat from the Optics Research Group of Delft University.

\section{References}

[1] R.Dandliker, K.Hug, J.Politch and E.Zimmermann, "High-accuracy distance measurements with multiple-wavelength interferometry" Opt. Eng. 34, 2407 (1995).

[2] B.L.Swinkels, N.Bhattacharya and J.J.M.Braat, "Correcting movement errors in frequency-sweeping interferometry" Opt. Lett. 30, 2242 (2005)
[3] K.Minoshima and H.Matsumoto, "High-accuracy measurement of $240 \mathrm{~m}$ distance in an optical tunnel by using of a compact femtosecond laser" Appl. Optics 39, 5512 (2000)

[4] Ki-Nam Joo and Seung-Woo Kim, "Absolute distance measurement by dispersive interferometry using a femtosecond pulse laser" Opt. Express 14, 5954 (2006)

[5] J. Ye, "Absolute measurement of a long, arbitrary distance to less than an optical fringe" Opt. Lett. 29, 1153 (2004).

[6] D.Jones, S.A.Diddams, J.K.Ranka, A.Stentz, R.S.Windeler, J.L.Hall and S.T.Cundiff, "Carrier-envelope phase control of femtosecond mode-locked lasers and direct optical frequency sysnthesis" Science 288, 635 (2000).

[7] R.Holzwarth, T.Udem, T.W. Hänsch, J.C. Knight, W.J. Wadsworth and P.S.J. Russell, "Optical frequency synthesizer for precision spectroscopy" Phys. Rev. Lett. 85, 2264 (2000).

[8] T.Yasui, K.Minoshima, and H.Matsumoto, "Stabilization of Femtosecond Mode-Locked Ti:Sapphire Laser for High-Accuracy Pulse Interferometry" IEEE J. Quantum Elect. 37, N0.1 (2001).

[9] K.P.Birch and M.J.Downs, "An Updated Edlen Equation for the Refractive Index of Air" Metrologia 30, 155 (1993).

[10] Y.Yamaoka, K.Minoshima, and H.Matsumoto, "Direct Measurement of the Group Refractive Index of Air with Interferometry between Adjacent Femtosecond Pulses" Appl. Optics 41, 4318 (2002). 